\title{
Variation in the Chemical Composition of Saccharina Japonica with Harvest Area and Culture Period
}

\author{
Jae-Ho Hwang', Nam-Gil Kim², Hee-Chul Woo ${ }^{3}$, Sung-Ju Rha1, Seon-Jae Kim ${ }^{4}$ and Tai-Sun Shin ${ }^{5 *}$ \\ ${ }^{1}$ College of Fisheries and Ocean Science, Chonnam National University, Yosu 550-749, Korea \\ ${ }^{2}$ Department Marine Biology and Aquaculture, Gyeongsang National University, 445 Inpyeong-dong, Tongyeong-si, Gyeongsangnam, 650-160, Korea \\ ${ }^{3}$ Department of Chemical Engineering, Pukyong National University, 365 Sinseon-ro, Yongdang-dong, Nam-gu, Busan, 608-739, Korea \\ ${ }^{4}$ Department of Marine Bio Food, Chonnam National University, Yeosu 550-749, Korea \\ ${ }^{5}$ Division of Food Nutrition Science, Chonnam National University, Gwangju 500-757, Korea
}

\begin{abstract}
Saccharina japonica is commercially important marine brown algae which grow as a single blade (reaching 10 meters in length) with a short stipe. In this study, the edible brown weed Sacchaina japonica was assessed for nutritional composition. Samples were collected monthly from seaweed farms at Kijang and Wando on the south coast of the Republic of Korea, during the 2011 culture season. S. japonica in Kijang and Wando showed the highest crude protein content in February and the highest carbohydrate content in July. Monthly changes in sugar, fatty acid, mineral, and total amino acid contents observed from February to July 2011. Fucose was the most abundant and galactose the second most abundant in the monosaccharide composition profiles, while mannose, glucose, xylose, ribose, and rhamnose were present in low quantities and lactose, mannitol, and arabinose were not detected. Significant increases of the major fatty acids in Kijang (C18:2 n-6 and C20:4 n-6) and Wando (C18:3 n-6) were observed as the culture period progressed. The highest mineral content of both Kijang and Wando samples is potassium and followed by sodium, calcium, magnesium, and so on. In the total amino acid contents, Kijang samples increased from February to April but decreased from May to July, while Wando samples increased on March but decreased from April to July.
\end{abstract}

Keywords: Saccharina japonica; Brown algae; Harvest area; Culture period; Chemical composition

\section{Introduction}

China, Japan, and the Republic of Korea are the largest consumers of edible seaweeds [1]. Seaweeds include high alginic acid, fucoidan, and laminara contents, so it is effective for hematocele and lipid metabolism improvement such as lowering blood pressure and cholesterol in the blood, and anti-cancer [2]. According to a survey conducted on worldwide production of aquatic plants, there are approximately 16 million tons of annual aquatic plants, of which 14.9 million tons produced by aquaculture [3]. Algal production in Korea is mainly limited to Porphyra tenera, Saccharina japonica, and Undaria pinnatifida, which comprise $94 \%$ of the total harvested seaweed [4]. $S$. japonica is very popular as a healthy food because of low calorie and abundant vitamin, mineral, dietary fiber, calcium, potassium, magnesium, phosphoric acid, and microelements and high iodine content as compared with other seaweeds [5].

In recent years, many studies on macro-algae have carried out and their proximate composition differs according to species, geographic origin, and seasonal conditions [6,7]. Growth change of laminaria closely related with culture period, most researchers studied to determine correlation between growth and nitrogen concentration [8]. Moreover, growth and chemical composition are various in different environments such as current, nutrients supply, fresh water inflow, and water temperature. Perennial Saccharina japonica generates alternately, and grows at subantarctic zone as well as temperate climate regions [9]. Cosson [10] reported that survival rate of Laminaria digitata spores is substantially lowered at over radiation intensity (about $170 \mu \mathrm{E} \cdot \mathrm{m}^{-2} \cdot \mathrm{s}^{-1}$ ). Kang and Koh [11] found that optimal growth temperature and light intensity of Laminaria japonica sporophyte were at $10^{\circ} \mathrm{C}$ and 70 $\mu \mathrm{E} \cdot \mathrm{m}^{-2} \cdot \mathrm{s}^{-1}$.

To our knowledge, detailed studies have not conducted to evaluate the effects of the culture period and harvest area on the chemical composition of $S$. japonica. This fundamental study performed to assess changes of proximate composition, sugar, fatty acid, mineral, and amino acid of $S$. japonica obtained from two sampling regions in Korea, Kijang and Wando, which had definitely different environment, and during the culture period from February to July.

\section{Materials and Methods}

\section{Sampling}

In order to observe variations in chemical composition during the harvest time, $S$. japonica was collected from an environmentally quite different seaweed farm at Kijang and Wando located on the southern coast of the Republic of Korea once a month from February to July 2011 (Figure 1). Both sporophytes of S. japonica transferred to the ocean at $0.5 \mathrm{~m}$ water depth in the same time (December 2010), and 5-20 individuals of whole $S$. japonica (blade, stem, and root) collected during the 2011 culture season. Freshly collected plants wrapped in paper towels with seawater, sealed in plastic bags, kept in an icebox, and transport to the laboratory where they washed with distilled water twice and freeze-dried. Each powered S. japonica (about $500 \mathrm{~g}$ ) used for triplicate analysis.

*Corresponding author: Tai-Sun Shin, Division of Food Nutrition Science Chonnam National University, Gwangju 500-757, Korea, Tel: +82-61-659-7415 Fax: +82-61-659-7415; E-mail: shints@chonnam.ac.kr

Received September 08, 2014; Accepted October 31, 2014; Published November 04, 2014

Citation: Hwang JH, Kim NG, Woo HC, Rha SJ, Kim SJ, et al. (2014) Variation in the Chemical Composition of Saccharina Japonica with Harvest Area and Culture Period. J Aquac Res Development 5: 286. doi:10.4172/2155-9546.1000286

Copyright: ( 2014 Hwang JH, et al. This is an open-access article distributed under the terms of the Creative Commons Attribution License, which permits unrestricted use, distribution, and reproduction in any medium, provided the original author and source are credited. 


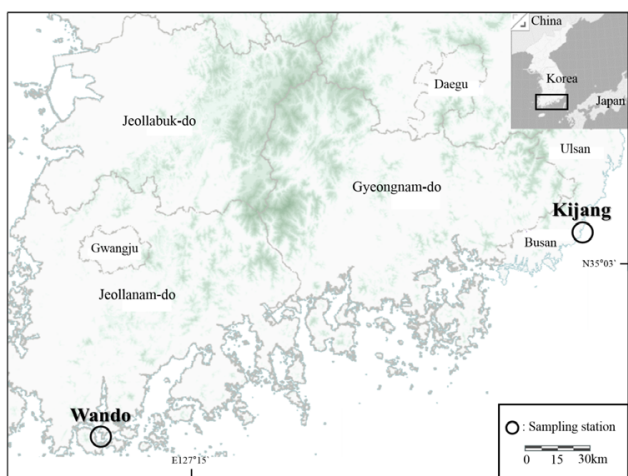

Figure 1: A map showing the site where Saccharide japonica were harvested during the 2011 culture period.

\begin{tabular}{|c|c|}
\hline & Condition \\
\hline Column & Shim-pack ISA-07 $(4.0 \mathrm{~mm} \times 250 \mathrm{~mm})$ \\
\hline Mobile phase & $\begin{array}{c}\text { A: potassium borate }(\mathrm{pH} 8.0) \\
\text { B: potassium borate }(\mathrm{pH} 9.0)\end{array}$ \\
\hline Flow rate & $0.6 \mathrm{~mL} / \mathrm{min}$, gradient \\
\hline Reagent & $1 \%$ arginine in $3 \%$ boric acid $(0.5 \mathrm{~mL})$ \\
\hline Reaction temperature & $150^{\circ} \mathrm{C}$ \\
\hline Detector & Fluorescence detector $(\mathrm{Ex}=320, \mathrm{Em}=430)$ \\
\hline Oven temperature & $65^{\circ} \mathrm{C}$ \\
\hline
\end{tabular}

Table 1: HPLC operating conditions for component sugars.

\section{General component analysis}

Moisture, crude protein, crude lipid, and ash content were determined using the standard methods described by the Association of Official Analytical Chemists [12]. Protein content analyzed using the semi-Kjeldahl method. Lipids extracted with anhydrous diethyl ether using a Soxhlet apparatus. Moisture quantified by oven drying the samples at $105^{\circ} \mathrm{C}$ for $12 \mathrm{~h}$. Ash was determined after incineration in a furnace at $550^{\circ} \mathrm{C}$. Total carbohydrate content calculated by subtracting the sum of moisture, crude protein, crude lipid, and ash mass from that of the total sample [13].

\section{Component sugar analysis}

In order to extract component sugar, a test sample $(100 \mathrm{mg})$ mixed in the $15 \mathrm{~mL}$ test tube with $5 \mathrm{~mL}$ of $2 \mathrm{M} \mathrm{HCl}$. The oxygen in the test tube replaced by nitrogen gas, sealed, and placed in a heating

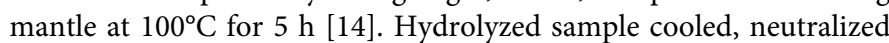
by adding $5 \mathrm{~mL}$ of $2 \mathrm{M} \mathrm{NaOH}$, and centrifuged at $650 \mathrm{~g}$ for $30 \mathrm{~min}$. $3 \mathrm{~mL}$ supernatant filtered through a Millipore membrane $(0.45 \mu \mathrm{m}$ pore size), and analyzed by operating conditions (Table 1) using HPLC (Prominence HPLC, Shimadzu Co, Ltd. Kyoto, Japan).

\section{Fatty acid composition analysis}

Bligh and Dyer extraction was performed using the following method [15]: Briefly, lipids were extracted from 5-g samples by homogenization with $100 \mathrm{~mL}$ of chloroform and $200 \mathrm{~mL}$ methanol. The samples were then filtered and evaporated to remove solvent. Fatty acid methyl esters (FAME) were prepared using boron trifluoride (BF3) according to a method described by the AOAC [12]. Quantitative analysis of FAME was carried out on a GC-2010 gas chromatograph(Shimadzu Co., Japan) equipped with a split/splitless capillary inlet system and a flame ionization detector (FID) using SP-
2560 capillary columns $(0.20-\mu \mathrm{m}$ stationary phase thickness, $100 \mathrm{~mm}$ (length) $\times 0.25 \mathrm{~mm}$ (i.d.); Supelco, Inc., USA). The sample $(0.5 \mu \mathrm{l})$ was injected in the split mode using an automatic injection system (AOC20i, Shimadzu Co., Japan). The oven temperature was programmed to increase from 160 to $220^{\circ} \mathrm{C}$ at $1^{\circ} \mathrm{C} \mathrm{min}{ }^{-1}$ with an initial hold of 5 min and final hold of $40 \mathrm{~min}$. The other operation parameters were as follows: injector temperature, $250^{\circ} \mathrm{C}$; detector temperature, $250^{\circ} \mathrm{C}$; helium carrier gas flow, $20 \mathrm{~cm} \mathrm{~s}^{-1}$; split ratio, 1:50. The peak areas for the calibration curves and for calculation of fatty acid composition of oil samples were measured using a GC Solution system (Shimadzu Co., Japan).

\section{Mineral contents}

For the determination of mineral elements (calcium, copper, iron, potassium, magnesium, manganese, sodium, and zinc), samples were digested by dry ashing and dissolved in $1 \mathrm{M} \mathrm{HCl} \mathrm{[12].} \mathrm{The} \mathrm{final}$ diluted solution for calcium contained 1\% lanthanum to overcome interferences. The concentration of the elements in S. japonica were determined with atomic absorption spectrophotometry (PerkinElmer, model 3110). Triplicate determinations for each element were carried out. The concentration of the elements were determined from calibration curves of the standard elements.

\section{Amino-acid analysis}

Samples $(0.5 \mathrm{~g})$ were acid-hydrolyzed with $3 \mathrm{~mL}$ of $6 \mathrm{~N} \mathrm{HCl}$ in vacuum-sealed hydrolysis vials at $121^{\circ} \mathrm{C}$ for $24 \mathrm{~h}$. Tubes were cooled after hydrolysis, opened, and placed in a rotary evaporator at $50^{\circ} \mathrm{C}$ to remove $\mathrm{HCl}$ from the sample. The residue was then adjusted to $\mathrm{pH} 2.2$ with $0.2 \mathrm{M}$ sodium citrate loading buffer $(\mathrm{pH} 2.2)$, diluted to a final volume of $10 \mathrm{~mL}$ with water, filtered through a Millipore membrane $(0.2 \mu \mathrm{m}$ pore size), and analyzed for amino acids using an amino-acid analyzer (Pharmacia Biochrom 20, Biochrom Ltd., UK).

\section{Statistical analysis}

All mean values were analyzed by one-way analysis of variance (ANOVA, SPSS 1999). Values are expressed as mean \pm standard deviation (SD; $\mathrm{n}=3$ replicates). Group means were considered to be significantly different at $\mathrm{p}<0.05$.

\section{Results}

\section{Changes in proximate composition with harvest area and culture period}

The proximate compositions of Kijang and Wando samples are shown in Tables 2 and 3 . There was a high variation in moisture, crude protein, ash and crude lipid content with culture period and harvest area among the Kijang and Wando samples collected at different months from February to July. S. japonica in Kijang and Wando showed the highest crude protein content in February and the highest carbohydrate content in July. In the crude lipid content, February samples in Kijang and Wando generally tended to decrease until July. There was a high variation in ash content with culture period and harvest area, ranging from $14.29 \pm 1.47 \%$ to $19.39 \pm 0.75 \%$ (Tables 2 and 3 ).

\section{Changes in component sugar and fatty acid composition with harvest area and culture period}

Component sugar compositions of Kijang and Wando samples are shown in Tables 4 and 5. Fucose was the most abundant and galactose the second most abundant in the monosaccharide composition profiles. Mannose, glucose, xylose, ribose, and rhamnose were present at low 
Citation: Hwang JH, Kim NG, Woo HC, Rha SJ, Kim SJ, et al. (2014) Variation in the Chemical Composition of Saccharina Japonica with Harvest Area and Culture Period. J Aquac Res Development 5: 286. doi:10.4172/2155-9546.1000286

Page 3 of 7

\begin{tabular}{|c|c|c|c|c|c|c|}
\hline \multirow{2}{*}{ Component } & \multicolumn{6}{|c|}{ Culture period } \\
\hline & Feb & Mar & Apr & May & Jun & Jul \\
\hline Moisture & $10.55 \pm 0.51^{a}$ & $10.67 \pm 0.45^{a}$ & $10.25 \pm 0.49^{a}$ & $10.31 \pm 0.98^{a}$ & $10.45 \pm 1.41^{\mathrm{a}}$ & $10.41 \pm 0.22^{a}$ \\
\hline Crude protein & $9.39 \pm 0.45^{\mathrm{a}}$ & $8.54 \pm 0.36^{b}$ & $7.61 \pm 0.34^{c}$ & $7.27 \pm 0.70^{\text {cd }}$ & $6.62 \pm 0.51^{d}$ & $5.72 \pm 0.51^{\mathrm{e}}$ \\
\hline Crude lipid & $1.69 \pm 0.08^{a}$ & $1.43 \pm 0.06^{b}$ & $1.35 \pm 0.06^{\mathrm{bc}}$ & $1.09 \pm 0.12^{\mathrm{d}}$ & $1.23 \pm 0.14^{\mathrm{cd}}$ & $1.17 \pm 0.02^{\mathrm{d}}$ \\
\hline Ash & $15.11 \pm 0.73^{b}$ & $17.88 \pm 0.72^{\mathrm{ab}}$ & $18.39 \pm 0.75^{a}$ & $17.86 \pm 2.58^{\mathrm{ab}}$ & $17.35 \pm 2.04^{\mathrm{ab}}$ & $16.51 \pm 0.34^{\mathrm{ab}}$ \\
\hline Carbohydrate $^{b}$ & $63.26 \pm 3.02^{\mathrm{a}}$ & $61.48 \pm 2.59^{a}$ & $62.40 \pm 2.61^{a}$ & $63.47 \pm 6.78^{a}$ & $64.35 \pm 5.62^{\mathrm{a}}$ & $66.19 \pm 1.23^{a}$ \\
\hline
\end{tabular}

a Values represent means \pm standard error $(n=3)$. Mean values in the same row followed by different letters differ significantly $(p<0.05)$. ${ }^{b} \mathrm{Carbohydrate}$ content $(\%)=100-(\%$ moisture $+\%$ protein $+\%$ lipid $+\%$ ash).

Table 2: Seasonal variation of proximate composition (\%) in the dried sea tangle (S. japonica) cultured at Kijang area

\begin{tabular}{|c|c|c|c|c|c|c|}
\hline \multirow{2}{*}{ Component } & \multicolumn{6}{|c|}{ Culture period } \\
\hline & Feb & Mar & Apr & May & Jun & Jul \\
\hline Moisture & $10.38 \pm 0.47^{a}$ & $10.51 \pm 0.45^{a}$ & $10.45 \pm 0.50^{\mathrm{a}}$ & $10.12 \pm 0.96^{a}$ & $10.34 \pm 1.18^{a}$ & $10.46 \pm 0.19^{a}$ \\
\hline Crude protein & $8.20 \pm 0.36^{a}$ & $8.20 \pm 0.40^{\mathrm{a}}$ & $7.51 \pm 0.31^{a}$ & $6.54 \pm 0.71^{b}$ & $5.58 \pm 0.80^{c}$ & $5.15 \pm 0.14^{c}$ \\
\hline Crude lipid & $2.00 \pm 0.09^{b}$ & $2.35 \pm 0.09^{a}$ & $1.56 \pm 0.07^{c}$ & $1.37 \pm 0.21^{\mathrm{cd}}$ & $1.26 \pm 0.10^{d}$ & $1.23 \pm 0.02^{\mathrm{d}}$ \\
\hline Ash & $16.68 \pm 0.77^{\mathrm{ab}}$ & $17.35 \pm 0.73^{a}$ & $17.86 \pm 0.80^{\mathrm{a}}$ & $15.82 \pm 2.39^{\mathrm{ab}}$ & $14.29 \pm 1.47^{b}$ & $14.69 \pm 0.29^{b}$ \\
\hline Carbohydrate $^{b}$ & $62.74 \pm 2.89^{a}$ & $61.59 \pm 2.61^{a}$ & $62.62 \pm 2.91^{a}$ & $66.15 \pm 7.29^{a}$ & $68.53 \pm 7.22^{a}$ & $68.47 \pm 0.49^{a}$ \\
\hline
\end{tabular}

${ }^{a}$ Values represent means \pm standard error $(n=3)$. Mean values in the same row followed by different letters differ significantly $(p<0.05)$. ${ }^{b} \mathrm{Carbohydrate}$ content $(\%)=100$ ( $\%$ moisture $+\%$ protein $+\%$ lipid $+\%$ ash $)$.

Table 3: Seasonal variation of proximate composition (\%) in the dried sea tangle (S. japonica) cultured at Wando area

\begin{tabular}{|c|c|c|c|c|c|c|}
\hline \multirow{2}{*}{ Sugar } & \multicolumn{6}{|c|}{ Culture period } \\
\hline & Feb & Mar & Apr & May & Jun & Jul \\
\hline Rhamnose & $0.11 \pm 0.00^{\mathrm{a}}$ & $0.08 \pm 0.00^{b}$ & $0.08 \pm 0.00^{\mathrm{b}}$ & $0.04 \pm 0.00^{c}$ & $0.04 \pm 0.00^{c}$ & $0.03 \pm 0.01^{\mathrm{d}}$ \\
\hline Ribose & $0.19 \pm 0.00^{a}$ & $0.10 \pm 0.00^{b}$ & $0.10 \pm 0.00^{\mathrm{b}}$ & $0.01 \pm 0.00^{d}$ & $0.02 \pm 0.00^{c}$ & $0.01 \pm 0.01^{\mathrm{d}}$ \\
\hline Mannose & $0.66 \pm 0.01^{b}$ & $0.55 \pm 0.01^{d}$ & $0.65 \pm 0.01^{b}$ & $0.72 \pm 0.02^{\mathrm{a}}$ & $0.60 \pm 0.01^{c}$ & $0.56 \pm 0.02^{\mathrm{d}}$ \\
\hline Fucose & $3.32 \pm 0.08^{c}$ & $4.17 \pm 0.13^{b}$ & $4.84 \pm 0.12^{\mathrm{a}}$ & $2.68 \pm 0.06^{d}$ & $2.27 \pm 0.06^{f}$ & $2.52 \pm 0.16^{e}$ \\
\hline Galactose & $2.31 \pm 0.06^{a}$ & $1.92 \pm 0.04^{b}$ & $1.97 \pm 0.06^{\mathrm{b}}$ & $0.80 \pm 0.02^{\mathrm{d}}$ & $0.88 \pm 0.02^{c}$ & $0.71 \pm 0.11^{\mathrm{e}}$ \\
\hline Xylose & $0.35 \pm 0.01^{a}$ & $0.24 \pm 0.01^{b}$ & $0.16 \pm 0.00^{c}$ & $0.06 \pm 0.00^{d}$ & $0.05 \pm 0.00^{e}$ & $0.05 \pm 0.00^{\mathrm{e}}$ \\
\hline Glucose & $0.55 \pm 0.01^{d}$ & $0.64 \pm 0.02^{c}$ & $0.68 \pm 0.02^{b}$ & $0.73 \pm 0.01^{a}$ & $0.72 \pm 0.02^{\mathrm{a}}$ & $0.53 \pm 0.12^{d}$ \\
\hline Total & $7.48 \pm 0.17^{b}$ & $7.70 \pm 0.17^{b}$ & $8.47 \pm 0.21^{\mathrm{a}}$ & $5.03 \pm 0.13^{c}$ & $4.56 \pm 0.11^{d}$ & $4.40 \pm 0.14^{\mathrm{d}}$ \\
\hline
\end{tabular}

aValues represent means \pm standard error $(n=3)$. Mean values in the same row followed by different letters differ significantly $(p<0.05)$.

Table 4: Seasonal variation of component sugar in the dried sea tangle (S. japonica) cultured at Kijang area ${ }^{a}$

\begin{tabular}{|c|c|c|c|c|c|c|}
\hline \multirow{2}{*}{ Sugar } & \multicolumn{6}{|c|}{ Culture period } \\
\hline & Feb & Mar & Apr & May & Jun & Jul \\
\hline Rhamnose & $0.12 \pm 0.00^{b}$ & $0.13 \pm 0.00^{\mathrm{a}}$ & $0.07 \pm 0.00^{c}$ & $0.03 \pm 0.00^{d}$ & $0.02 \pm 0.00^{\mathrm{e}}$ & $0.02 \pm 0.00^{\mathrm{e}}$ \\
\hline Ribose & $0.19 \pm 0.01^{\mathrm{a}}$ & $0.10 \pm 0.00^{\mathrm{b}}$ & $0.09 \pm 0.00^{c}$ & $0.01 \pm 0.00^{e}$ & $0.02 \pm 0.00^{d}$ & $0.01 \pm 0.01^{\mathrm{e}}$ \\
\hline Mannose & $0.75 \pm 0.02^{\mathrm{a}}$ & $0.67 \pm 0.02^{c}$ & $0.74 \pm 0.02^{\mathrm{a}}$ & $0.70 \pm 0.02^{b}$ & $0.59 \pm 0.01^{d}$ & $0.47 \pm 0.08^{e}$ \\
\hline Fucose & $3.49 \pm 0.09^{a}$ & $2.98 \pm 0.06^{b}$ & $2.92 \pm 0.08^{b c}$ & $3.01 \pm 0.07^{b}$ & $2.83 \pm 0.05^{c}$ & $1.85 \pm 0.54^{d}$ \\
\hline Galactose & $2.21 \pm 0.05^{\mathrm{a}}$ & $1.65 \pm 0.04^{c}$ & $1.81 \pm 0.04^{b}$ & $0.80 \pm 0.02^{d}$ & $0.80 \pm 0.01^{d}$ & $0.63 \pm 0.09^{\mathrm{e}}$ \\
\hline Xylose & $0.43 \pm 0.01^{\mathrm{a}}$ & $0.28 \pm 0.01^{c}$ & $0.30 \pm 0.01^{b}$ & $0.06 \pm 0.00^{d}$ & $0.07 \pm 0.00^{d}$ & $0.05 \pm 0.01^{\mathrm{e}}$ \\
\hline Glucose & $0.67 \pm 0.02^{b}$ & $0.67 \pm 0.02^{b}$ & $0.73 \pm 0.02^{\mathrm{a}}$ & $0.72 \pm 0.01^{\mathrm{a}}$ & $0.42 \pm 0.01^{c}$ & $0.42 \pm 0.01^{c}$ \\
\hline Total & $7.87 \pm 0.21^{\mathrm{a}}$ & $6.48 \pm 0.14^{b}$ & $6.65 \pm 0.21^{b}$ & $5.34 \pm 0.16^{c}$ & $4.76 \pm 0.11^{d}$ & $3.45 \pm 0.73^{e}$ \\
\hline
\end{tabular}

avalues represent means \pm standard error $(n=3)$. Mean values in the same row followed by different letters differ significantly $(p<0.05)$.

Table 5: Seasonal variation of component sugar in the dried sea tangle (S. japonica) cultured at Wando area ${ }^{a}$

quantities, and lactose, mannitol, and arabinose were not detected.

The fatty acid compositions of Kijang and Wando samples are shown in Tables 6 and 7. Lignoceric acid (24:0) was the most abundant fatty acid, followed by arachidonic acid (20:4 n-6), oleic acid (18:1 n-9), and palmitic acid (16:0). Polyunsaturated fatty acid (PUFA) and monounsaturated fatty acid (MUFA) constituted about $54.9 \%$, $52.3 \%$ of total fatty acids, and saturated fatty acids (SFA) represented $45.1 \%, 47.7 \%$ of the total fatty acids in the Kijang and Wando samples, respectively. The Kijang-Jul samples showed the highest PUFA composition (37.5\%) among the samples, while Wando-Mar showed the lowest PUFA composition (30.1\%), indicating that there was a high variation in fatty acid contents with the harvest area and culture period.

\section{Changes in mineral content and total amino acid composition with harvest area and culture period}

The mineral contents of Kijang and Wando samples are shown in Tables 8 and 9. The results show that $S$. japonica is rich in $\mathrm{K}$ and $\mathrm{Na}$ with moderate amounts of $\mathrm{Ca}$ and $\mathrm{Mg}$ whereas $\mathrm{Cu}, \mathrm{Fe}, \mathrm{Mn}$, and $\mathrm{Zn}$ are present in small quantities. The total amino acid (TAA) compositions of Kijang and Wando samples are shown in Tables 10 and 11. Glutamic acid, aspartic acid, alanine, and leucine were the most common amino acids in all samples, while the percentage of cysteine was the lowest in the TAA profile. TAA of Kijang samples decreased during the harvest time from April to July while TAA of Wando samples decreased from March to July. 
Citation: Hwang JH, Kim NG, Woo HC, Rha SJ, Kim SJ, et al. (2014) Variation in the Chemical Composition of Saccharina Japonica with Harvest Area and Culture Period. J Aquac Res Development 5: 286. doi:10.4172/2155-9546.1000286

Page 4 of 7

\begin{tabular}{|c|c|c|c|c|c|c|}
\hline \multirow{2}{*}{$\begin{array}{l}\text { Fatty acid } \\
(\%)\end{array}$} & \multicolumn{6}{|c|}{ Culture period } \\
\hline & Feb & Mar & Apr & May & Jun & Jul \\
\hline $12: 0$ & $0.18 \pm 0.01^{c}$ & $0.27 \pm 0.01^{a}$ & $0.21 \pm 0.00^{b}$ & $0.03 \pm 0.00^{f}$ & $0.05 \pm 0.00^{e}$ & $0.07 \pm 0.00^{d}$ \\
\hline $14: 0$ & $9.50 \pm 0.21^{a}$ & $9.66 \pm 0.19^{a}$ & $7.11 \pm 0.15^{c}$ & $4.73 \pm 0.15^{\mathrm{e}}$ & $7.68 \pm 0.15^{b}$ & $6.02 \pm 0.12^{d}$ \\
\hline 16:0 & $13.53 \pm 0.31^{d}$ & $16.25 \pm 0.35^{b}$ & $17.22 \pm 0.22^{a}$ & $17.34 \pm 0.40^{\mathrm{a}}$ & $14.51 \pm 0.33^{c}$ & $11.07 \pm 0.27^{e}$ \\
\hline $16: 1 n-7$ & $3.08 \pm 0.08^{c}$ & $3.78 \pm 0.12^{\mathrm{a}}$ & $3.44 \pm 0.08^{b}$ & $3.06 \pm 0.07^{c}$ & $3.49 \pm 0.09^{b}$ & $3.20 \pm 0.09^{c}$ \\
\hline 18:0 & $0.86 \pm 0.02^{\circ}$ & $1.00 \pm 0.02^{b}$ & $1.16 \pm 0.03^{a}$ & $0.69 \pm 0.02^{d}$ & $0.87 \pm 0.02^{c}$ & $0.89 \pm 0.02^{c}$ \\
\hline $18: 1 n-9$ & $19.20 \pm 0.40^{a}$ & $16.83 \pm 0.42^{\mathrm{c}}$ & $15.67 \pm 0.42^{d}$ & $13.30 \pm 0.40^{\mathrm{e}}$ & $16.27 \pm 0.39^{c d}$ & $17.75 \pm 0.42^{b}$ \\
\hline $18: 2 n-6$ & $5.58 \pm 0.15^{b}$ & $6.79 \pm 0.19^{a}$ & $6.78 \pm 0.15^{a}$ & $6.93 \pm 0.14^{a}$ & $6.95 \pm 0.16^{a}$ & $7.03 \pm 0.16^{a}$ \\
\hline $18: 3 n-6$ & $1.78 \pm 0.04^{e}$ & $2.17 \pm 0.05^{d}$ & $2.90 \pm 0.07^{c}$ & $2.94 \pm 0.07^{c}$ & $3.97 \pm 0.09^{a}$ & $3.76 \pm 0.09^{b}$ \\
\hline $18: 3 n-3$ & $7.38 \pm 0.15^{a}$ & $6.54 \pm 0.16^{c}$ & $6.79 \pm 0.21^{b}$ & $3.84 \pm 0.09^{d}$ & $2.96 \pm 0.07^{e}$ & $2.97 \pm 0.06^{\mathrm{e}}$ \\
\hline $20: 0$ & $0.40 \pm 0.01^{c}$ & $0.50 \pm 0.01^{b}$ & $0.50 \pm 0.01^{b}$ & $0.26 \pm 0.01^{d}$ & $0.51 \pm 0.01^{\mathrm{ab}}$ & $0.53 \pm 0.01^{\mathrm{a}}$ \\
\hline $20: 2 n-6$ & $1.30 \pm 0.03^{c}$ & $1.76 \pm 0.04^{a}$ & $1.59 \pm 0.05^{b}$ & $1.66 \pm 0.04^{b}$ & $1.80 \pm 0.04^{\mathrm{a}}$ & $1.78 \pm 0.04^{\mathrm{a}}$ \\
\hline $20: 3 n-6$ & $1.42 \pm 0.03^{e}$ & $1.95 \pm 0.04^{\mathrm{a}}$ & $1.84 \pm 0.05^{b}$ & $1.67 \pm 0.04^{c}$ & $1.53 \pm 0.03^{d}$ & $1.62 \pm 0.05^{c}$ \\
\hline $20: 4 n-6$ & $13.35 \pm 0.30^{d}$ & $14.92 \pm 0.40^{c}$ & $15.37 \pm 0.32^{c}$ & $19.19 \pm 0.45^{b}$ & $19.40 \pm 0.21^{b}$ & $20.34 \pm 0.45^{a}$ \\
\hline $\mathrm{C} 24: 0$ & $22.44 \pm 0.44^{\mathrm{b}}$ & $17.58 \pm 0.40^{d}$ & $19.42 \pm 0.54^{c}$ & $24.36 \pm 0.64^{a}$ & $20.01 \pm 0.21^{\mathrm{c}}$ & $22.97 \pm 0.54^{b}$ \\
\hline Saturates & $46.91 \pm 1.07^{\mathrm{ab}}$ & $45.26 \pm 1.29^{b c}$ & $45.62 \pm 1.11^{\mathrm{abc}}$ & $47.42 \pm 0.62^{\mathrm{a}}$ & $43.64 \pm 0.93^{c}$ & $41.55 \pm 1.26^{d}$ \\
\hline Monoenes & $22.27 \pm 0.59^{a}$ & $20.62 \pm 0.46^{b c}$ & $19.11 \pm 0.61^{d}$ & $16.35 \pm 0.49^{e}$ & $19.77 \pm 0.45^{\mathrm{cd}}$ & $20.95 \pm 0.48^{b}$ \\
\hline Polyenes & $30.81 \pm 0.67^{d}$ & $34.13 \pm 0.80^{c}$ & $35.27 \pm 0.76^{b c}$ & $36.23 \pm 0.81^{a b}$ & $36.60 \pm 1.02^{\mathrm{ab}}$ & $37.50 \pm 0.45^{a}$ \\
\hline $\mathrm{P} / \mathrm{S}$ & $0.66 \pm 0.02^{d}$ & $0.75 \pm 0.02^{c}$ & $0.77 \pm 0.02^{c}$ & $0.76 \pm 0.02^{c}$ & $0.84 \pm 0.02^{b}$ & $0.90 \pm 0.02^{\mathrm{a}}$ \\
\hline
\end{tabular}

aValues represent means \pm standard error $(n=3)$. Mean values in the same row followed by different letters differ significantly $(p<0.05)$.

Table 6: Seasonal variation of fatty acid composition (percentage of weight) in the dried sea tangle (S. japonica) cultured at Kijang area ${ }^{\text {. }}$

\begin{tabular}{|c|c|c|c|c|c|c|}
\hline \multirow{2}{*}{$\begin{array}{l}\text { Fatty acid } \\
(\%)\end{array}$} & \multicolumn{6}{|c|}{ Culture period } \\
\hline & Feb & Mar & Apr & May & Jun & Jul \\
\hline $12: 0$ & $0.28 \pm 0.01^{b}$ & $0.58 \pm 0.01^{a}$ & $0.09 \pm 0.00^{d}$ & $0.04 \pm 0.00^{e}$ & $0.16 \pm 0.00^{c}$ & $0.08 \pm 0.00^{d}$ \\
\hline 14:0 & $10.47 \pm 0.25^{b}$ & $10.92 \pm 0.13^{a}$ & $8.70 \pm 0.17^{c}$ & $6.72 \pm 0.18^{e}$ & $7.09 \pm 0.15^{d}$ & $5.74 \pm 0.18^{f}$ \\
\hline 16:0 & $16.08 \pm 0.40^{c}$ & $16.94 \pm 0.33^{b}$ & $17.13 \pm 0.45^{b}$ & $18.07 \pm 0.37^{a}$ & $11.25 \pm 0.14^{d}$ & $10.58 \pm 0.24^{e}$ \\
\hline $16: 1 n-7$ & $2.92 \pm 0.07^{\mathrm{d}}$ & $3.10 \pm 0.08^{c}$ & $3.45 \pm 0.07^{b}$ & $3.15 \pm 0.05^{c}$ & $3.79 \pm 0.09^{a}$ & $3.20 \pm 0.07^{c}$ \\
\hline 18:0 & $1.25 \pm 0.02^{\mathrm{a}}$ & $1.14 \pm 0.02^{\mathrm{b}}$ & $0.82 \pm 0.01^{c}$ & $0.81 \pm 0.02^{c}$ & $0.82 \pm 0.02^{c}$ & $0.81 \pm 0.02^{c}$ \\
\hline $18: 1 \mathrm{n}-9$ & $17.26 \pm 0.19^{a}$ & $17.07 \pm 0.34^{a}$ & $14.95 \pm 0.30^{c}$ & $15.41 \pm 0.43^{b c}$ & $15.71 \pm 0.42^{b}$ & $15.95 \pm 0.48^{b}$ \\
\hline $18: 2 n-6$ & $5.24 \pm 0.05^{d}$ & $5.46 \pm 0.17^{d}$ & $6.03 \pm 0.17^{c}$ & $6.38 \pm 0.13^{b}$ & $6.86 \pm 0.15^{\mathrm{a}}$ & $6.51 \pm 0.13^{b}$ \\
\hline $18: 3 n-6$ & $1.75 \pm 0.04^{f}$ & $2.22 \pm 0.05^{\mathrm{e}}$ & $2.34 \pm 0.06^{d}$ & $2.65 \pm 0.07^{c}$ & $3.05 \pm 0.08^{b}$ & $3.67 \pm 0.09^{a}$ \\
\hline $18: 3 n-3$ & $6.85 \pm 0.16^{a}$ & $5.70 \pm 0.13^{c}$ & $6.26 \pm 0.14^{b}$ & $4.70 \pm 0.13^{d}$ & $4.15 \pm 0.13^{e}$ & $4.00 \pm 0.09^{e}$ \\
\hline $20: 0$ & $0.40 \pm 0.01^{d}$ & $0.47 \pm 0.01^{b}$ & $0.43 \pm 0.01^{c}$ & $0.35 \pm 0.00^{e}$ & $0.42 \pm 0.01^{c}$ & $0.49 \pm 0.01^{a}$ \\
\hline $20: 2 n-6$ & $1.49 \pm 0.04^{b}$ & $1.71 \pm 0.03^{a}$ & $1.40 \pm 0.04^{c}$ & $1.68 \pm 0.05^{\mathrm{a}}$ & $1.26 \pm 0.04^{d}$ & $1.52 \pm 0.04^{b}$ \\
\hline $20: 3 n-6$ & $1.58 \pm 0.03^{b}$ & $1.77 \pm 0.04^{a}$ & $1.63 \pm 0.04^{b}$ & $1.51 \pm 0.03^{c}$ & $1.59 \pm 0.04^{b}$ & $1.49 \pm 0.03^{c}$ \\
\hline $20: 4 n-6$ & $13.55 \pm 0.32^{\mathrm{e}}$ & $13.19 \pm 0.41^{\mathrm{e}}$ & $16.82 \pm 0.39^{c}$ & $15.60 \pm 0.37^{d}$ & $17.55 \pm 0.36^{b}$ & $18.96 \pm 0.45^{a}$ \\
\hline C24:0 & $20.89 \pm 0.60^{c}$ & $19.73 \pm 0.45^{\mathrm{d}}$ & $19.95 \pm 0.50^{\text {cd }}$ & $22.93 \pm 0.24^{b}$ & $26.30 \pm 0.74^{a}$ & $27.00 \pm 0.71^{a}$ \\
\hline Saturates & $49.37 \pm 1.56^{a}$ & $49.78 \pm 0.98^{a}$ & $47.12 \pm 1.16^{b}$ & $48.93 \pm 1.04^{a}$ & $46.04 \pm 1.12^{\mathrm{bc}}$ & $44.71 \pm 0.58^{c}$ \\
\hline Monoenes & $20.17 \pm 0.46^{a}$ & $20.17 \pm 0.46^{a}$ & $18.40 \pm 0.32^{c}$ & $18.56 \pm 0.43^{c}$ & $19.50 \pm 0.62^{\mathrm{ab}}$ & $19.15 \pm 0.57^{b c}$ \\
\hline Polyenes & $30.46 \pm 0.69^{d}$ & $30.05 \pm 0.80^{d}$ & $34.48 \pm 0.62^{\mathrm{b}}$ & $32.51 \pm 0.90^{c}$ & $34.46 \pm 0.74^{b}$ & $36.14 \pm 0.81^{a}$ \\
\hline $\mathrm{P} / \mathrm{S}$ & $0.62 \pm 0.01^{d}$ & $0.60 \pm 0.02^{d}$ & $0.73 \pm 0.02^{b}$ & $0.66 \pm 0.01^{c}$ & $0.75 \pm 0.02^{b}$ & $0.81 \pm 0.02^{a}$ \\
\hline
\end{tabular}

aValues represent means \pm standard error $(n=3)$. Mean values in the same row followed by different letters differ significantly $(p<0.05)$.

Table 7: Seasonal variation of fatty acid composition (percentage of weight) in the dried sea tangle (S. japonica) cultured at Wando area ${ }^{\text {. }}$

\section{Discussion}

There are big environmental differences between Wando and Kijang. Wando is semi-closed sea, and affected by big tide and fresh water inflow from many rivers around. Kijang has a small tide, but high temperature high salinity Tsushima current and low temperature low salinity North Korea current meets in this area. To our knowledge, this is the first study that evaluated differences in the nutritional composition of $S$. japonica with harvest area and culture period. We found that protein content of $S$. japonica was highest in February and the carbohydrate content was highest in July for the Kijang and Wando samples over the culture period from February to July 2011. A similar pattern was previously reported for the collection of Laminaria japonica [16]. Rosemberg and Ramus [17] found inverse relationships between carbohydrate and protein content in the red seaweed Gracilaria cervicornis during collection from July 2000 to June 2001. The seaweed protein content was lowest when photosynthetic activity and carbohydrate synthesis were highest. Shin et al. $[18,19]$ found that carbohydrate content of Porphyra yezoensis increased with late culture period: Dec (39.4\%), Feb (47.2\%). However, the protein content decreased with late culture period: Dec (39.4\%), Feb (34.6\%). Lipid content was not affected by culture period. A positive correlation was also detected between carbohydrate and temperature, along with correlations with salinity and solar radiation, which indicated that carbohydrate synthesis and protein concentration are affected by several seasonal factors, including water temperature, nitrogen content, and light intensity $[16,18]$. The lipid content was low relative to the other chemical constituents. However, the lipid content observed 
Citation: Hwang JH, Kim NG, Woo HC, Rha SJ, Kim SJ, et al. (2014) Variation in the Chemical Composition of Saccharina Japonica with Harvest Area and Culture Period. J Aquac Res Development 5: 286. doi:10.4172/2155-9546.1000286

Page 5 of 7

\begin{tabular}{|c|c|c|c|c|c|c|}
\hline \multirow{2}{*}{ Mineral } & \multicolumn{7}{|c|}{ Culture period } \\
\cline { 2 - 7 } & Feb & Mar & Apr & May & Jun & Jul \\
\hline $\mathrm{Ca}$ & $567.11 \pm 16.71^{\mathrm{e}}$ & $972.86 \pm 23.27^{\mathrm{a}}$ & $858.81 \pm 16.54^{\mathrm{b}}$ & $745.45 \pm 21.30^{\mathrm{d}}$ & $783.84 \pm 17.89^{\mathrm{c}}$ & $741.41 \pm 18.08^{\mathrm{d}}$ \\
\hline $\mathrm{Cu}$ & $0.29 \pm 0.01^{\mathrm{e}}$ & $0.46 \pm 0.01^{\mathrm{b}}$ & $0.34 \pm 0.01^{\mathrm{c}}$ & $0.47 \pm 0.01^{\mathrm{b}}$ & $0.67 \pm 0.01^{\mathrm{a}}$ & $0.31 \pm 0.01^{\mathrm{d}}$ \\
\hline $\mathrm{Fe}$ & $8.15 \pm 0.18^{\mathrm{a}}$ & $3.70 \pm 0.08^{\mathrm{d}}$ & $3.16 \pm 0.04^{\mathrm{a}}$ & $6.20 \pm 0.14^{\mathrm{b}}$ & $3.39 \pm 0.08^{\mathrm{e}}$ & $4.46 \pm 0.09^{\mathrm{c}}$ \\
\hline $\mathrm{K}$ & $3325.83 \pm 83.43^{\mathrm{c}}$ & $3516.51 \pm 111.07^{\mathrm{b}}$ & $4158.54 \pm 99.29^{\mathrm{a}}$ & $3554.55 \pm 80.98^{\mathrm{b}}$ & $3578.28 \pm 95.21^{\mathrm{b}}$ & $3165.47 \pm 67.11^{\mathrm{d}}$ \\
\hline $\mathrm{Mg}$ & $630.63 \pm 17.51^{\mathrm{c}}$ & $592.81 \pm 11.93^{\mathrm{d}}$ & $606.55 \pm 17.32^{\mathrm{cd}}$ & $887.89 \pm 20.04^{\mathrm{a}}$ & $821.58 \pm 17.91^{\mathrm{b}}$ & $794.79 \pm 12.45^{\mathrm{b}}$ \\
\hline $\mathrm{Mn}$ & $0.44 \pm 0.01^{\mathrm{d}}$ & $0.70 \pm 0.02^{\mathrm{b}}$ & $0.69 \pm 0.02^{\mathrm{b}}$ & $0.68 \pm 0.02^{\mathrm{b}}$ & $0.86 \pm 0.02^{\mathrm{a}}$ & $0.55 \pm 0.01^{\mathrm{c}}$ \\
\hline $\mathrm{Na}$ & $1209.21 \pm 32.09^{\mathrm{d}}$ & $1440.73 \pm 39.94^{\mathrm{a}}$ & $1361.45 \pm 30.09^{\mathrm{b}}$ & $1285.19 \pm 26.22^{\mathrm{c}}$ & $1253.65 \pm 28.78^{\mathrm{cd}}$ & $1204.93 \pm 29.30^{\mathrm{d}}$ \\
\hline $\mathrm{Zn}$ & $1.65 \pm 0.04^{\mathrm{a}}$ & $2.34 \pm 0.05^{\mathrm{c}}$ & $2.19 \pm 0.05^{\mathrm{d}}$ & $2.76 \pm 0.07^{\mathrm{b}}$ & $0.37 \pm 0.01^{\mathrm{f}}$ & $3.04 \pm 0.04^{\mathrm{a}}$ \\
\hline Total & $5,743.31 \pm 120.58^{\mathrm{c}}$ & $6,530.11 \pm 156.09^{\mathrm{b}}$ & $6,991.73 \pm 211.17^{\mathrm{a}}$ & $6,483.19 \pm 154.05^{\mathrm{b}}$ & $6,442.64 \pm 151.48^{\mathrm{b}}$ & $5,914.96 \pm 126.98^{\mathrm{c}}$ \\
\hline
\end{tabular}

aValues represent means \pm standard error $(n=3)$. Mean values in the same row followed by different letters differ significantly $(p<0.05)$.

Table 8: Seasonal variation of mineral contents in the dried sea tangle (S. japonica) cultured at Kijang area ${ }^{a}$.

\begin{tabular}{|c|c|c|c|c|c|c|}
\hline \multirow{2}{*}{ Mineral } & \multicolumn{6}{|c|}{ Culture period } \\
\hline & Feb & Mar & Apr & May & Jun & Jul \\
\hline $\mathrm{Ca}$ & $900.91 \pm 22.15^{\mathrm{a}}$ & $913.15 \pm 17.93^{a}$ & $913.58 \pm 23.49^{a}$ & $789.90 \pm 18.30^{b}$ & $730.30 \pm 13.90^{c}$ & $771.72 \pm 14.14^{b}$ \\
\hline $\mathrm{Cu}$ & $0.56 \pm 0.01^{c}$ & $0.40 \pm 0.01^{d}$ & $0.94 \pm 0.02^{a}$ & $0.90 \pm 0.02^{\mathrm{b}}$ & $0.22 \pm 0.00^{\mathrm{e}}$ & $0.12 \pm 0.00^{f}$ \\
\hline $\mathrm{Fe}$ & $2.89 \pm 0.06^{\mathrm{e}}$ & $2.48 \pm 0.06^{f}$ & $3.55 \pm 0.10^{d}$ & $5.52 \pm 0.15^{\mathrm{a}}$ & $4.92 \pm 0.05^{c}$ & $5.24 \pm 0.15^{b}$ \\
\hline $\mathrm{K}$ & $3683.32 \pm 84.26^{c}$ & $4020.03 \pm 114.39^{a}$ & $3847.32 \pm 93.92^{b}$ & $3643.64 \pm 47.59^{c}$ & $3260.86 \pm 69.51^{d}$ & $3182.01 \pm 41.93^{d}$ \\
\hline $\mathrm{Mg}$ & $644.72 \pm 17.15^{d}$ & $723.22 \pm 15.96^{c}$ & $593.00 \pm 18.77^{e}$ & $836.84 \pm 25.04^{\mathrm{ab}}$ & $852.96 \pm 19.57^{a}$ & $820.82 \pm 17.93^{b}$ \\
\hline $\mathrm{Mn}$ & $0.77 \pm 0.02^{\mathrm{a}}$ & $0.62 \pm 0.01^{d}$ & $0.73 \pm 0.02^{\mathrm{b}}$ & $0.47 \pm 0.01^{\mathrm{e}}$ & $0.65 \pm 0.02^{c}$ & $0.31 \pm 0.00^{f}$ \\
\hline $\mathrm{Na}$ & $1378.52 \pm 32.66^{b}$ & $1435.11 \pm 43.64^{b}$ & $1613.96 \pm 36.48^{a}$ & $1173.17 \pm 25.60^{d}$ & $1141.14 \pm 33.21^{d}$ & $1286.39 \pm 25.75^{c}$ \\
\hline $\mathrm{Zn}$ & $1.44 \pm 0.03^{f}$ & $2.13 \pm 0.06^{d}$ & $2.35 \pm 0.05^{c}$ & $2.53 \pm 0.08^{b}$ & $3.04 \pm 0.06^{a}$ & $1.69 \pm 0.05^{e}$ \\
\hline Total & $6613.13 \pm 165.73^{b}$ & $7097.14 \pm 138.26^{a}$ & $6975.43 \pm 183.16^{a}$ & $6452.97 \pm 132.95^{b}$ & $5,994.09 \pm 84.62^{c}$ & $6068.3 \pm 96.61^{c}$ \\
\hline
\end{tabular}

aValues represent means \pm standard error $(n=3)$. Mean values in the same row followed by different letters differ significantly $(p<0.05)$.

Table 9: Seasonal variation of mineral contents in the dried sea tangle (S. japonica) cultured at Wando area ${ }^{\mathrm{a}}$.

\begin{tabular}{|c|c|c|c|c|c|c|}
\hline \multirow{2}{*}{ Amino acid } & \multicolumn{6}{|c|}{ Culture period } \\
\hline & Feb & Mar & Apr & May & Jun & Jul \\
\hline Aspartic acid & $1574.47 \pm 46.38^{a}$ & $1422.98 \pm 34.04^{b}$ & $1415.36 \pm 27.26^{b}$ & $1250.12 \pm 35.73^{c}$ & $1087.68 \pm 21.32^{d}$ & $1032.76 \pm 25.39^{d}$ \\
\hline Threonine $^{*}$ & $693.70 \pm 15.40^{a}$ & $721.08 \pm 14.38^{a}$ & $718.71 \pm 15.30^{a}$ & $636.35 \pm 20.0^{\mathrm{b}}$ & $526.62 \pm 14.11^{c}$ & $446.02 \pm 9.84^{d}$ \\
\hline Serine & $773.96 \pm 17.51^{a}$ & $677.69 \pm 14.53^{b}$ & $674.86 \pm 8.57^{\mathrm{b}}$ & $604.14 \pm 13.78^{c}$ & $515.94 \pm 3.45^{d}$ & $487.02 \pm 9.61^{d}$ \\
\hline Glutamic acid & $1718.74 \pm 43.11^{a}$ & $1623.86 \pm 51.29^{b}$ & $1615.31 \pm 38.57^{b}$ & $1502.92 \pm 34.24^{c}$ & $1223.20 \pm 9.64^{d}$ & $1089.29 \pm 23.09^{e}$ \\
\hline Proline & $676.85 \pm 18.80^{a}$ & $565.19 \pm 11.37^{b}$ & $562.15 \pm 16.05^{b}$ & $542.35 \pm 12.24^{b}$ & $497.45 \pm 9.05^{c}$ & $400.25 \pm 6.27^{d}$ \\
\hline Glycine & $895.55 \pm 18.66^{a}$ & $821.67 \pm 20.32^{b}$ & $817.98 \pm 21.71^{b}$ & $765.58 \pm 22.93^{c}$ & $622.11 \pm 8.24^{d}$ & $536.65 \pm 14.07^{e}$ \\
\hline Alanine & $1105.96 \pm 29.35^{a}$ & $995.41 \pm 27.59^{b}$ & $990.79 \pm 21.90^{b}$ & $724.24 \pm 14.78^{c}$ & $602.70 \pm 10.81^{e}$ & $666.54 \pm 16.21^{d}$ \\
\hline Cystine & N.D. & N.D. & N.D. & N.D. & N.D. & N.D. \\
\hline Valine* & $592.06 \pm 12.43^{c}$ & $773.56 \pm 18.45^{a}$ & $769.75 \pm 24.25^{a}$ & $694.82 \pm 15.83^{b}$ & $557.45 \pm 0.26^{d}$ & $384.18 \pm 10.56^{\mathrm{e}}$ \\
\hline Methionine & $345.06 \pm 10.97^{a}$ & $321.79 \pm 7.45^{b}$ & $319.66 \pm 7.33^{b}$ & $275.21 \pm 5.95^{c}$ & $210.27 \pm 2.02^{d}$ & $188.26 \pm 3.41^{\mathrm{e}}$ \\
\hline Isoleucine* & $435.32 \pm 9.14^{d}$ & $623.87 \pm 14.91^{a}$ & $620.55 \pm 18.74^{a}$ & $586.15 \pm 13.93^{b}$ & $496.92 \pm 4.37^{\circ}$ & $424.00 \pm 9.11^{d}$ \\
\hline Leucine $^{*}$ & $1094.16 \pm 26.91^{b}$ & $1174.31 \pm 23.05^{\mathrm{a}}$ & $1168.03 \pm 30.03^{a}$ & $997.35 \pm 23.10^{c}$ & $842.37 \pm 5.94^{d}$ & $656.88 \pm 12.04^{e}$ \\
\hline Tyrosine* & $366.60 \pm 8.37^{a}$ & $299.66 \pm 8.00^{b}$ & $297.48 \pm 6.15^{b}$ & $285.19 \pm 6.73^{c}$ & $272.69 \pm 2.88^{d}$ & $222.90 \pm 5.42^{\mathrm{e}}$ \\
\hline Phenylalanine* & $636.80 \pm 12.54^{b}$ & $697.82 \pm 15.93^{a}$ & $693.92 \pm 19.42^{\mathrm{a}}$ & $585.45 \pm 15.41^{c}$ & $512.50 \pm 4.42^{\mathrm{d}}$ & $393.85 \pm 11.04^{\mathrm{e}}$ \\
\hline Histidine & $325.11 \pm 7.44^{c}$ & $394.50 \pm 11.23^{a}$ & $394.62 \pm 9.63^{a}$ & $342.34 \pm 4.47^{b}$ & $304.46 \pm 3.28^{d}$ & $279.06 \pm 3.68^{e}$ \\
\hline Lysine* & $653.32 \pm 17.38^{b}$ & $700.03 \pm 15.45^{a}$ & $696.74 \pm 22.06^{a}$ & $513.45 \pm 15.36^{c}$ & $480.60 \pm 6.28^{d}$ & $430.39 \pm 9.40^{e}$ \\
\hline Arginine & $511.63 \pm 11.16^{c}$ & $660.01 \pm 15.48^{a}$ & $658.02 \pm 14.11^{a}$ & $641.75 \pm 14.39^{a}$ & $544.90 \pm 3.66^{b}$ & $482.10 \pm 5.36^{d}$ \\
\hline Total & $12399.29 \pm 293.74^{a}$ & $12473.43 \pm 379.31^{a}$ & $12413.91 \pm 280.61^{a}$ & $9986.94 \pm 217.90^{b}$ & $9217.87 \pm 129.32^{c}$ & $7783.17 \pm 155.80^{d}$ \\
\hline
\end{tabular}

aValues represent means \pm standard error $(n=3)$. Mean values in the same row followed by different letters differ significantly $(p<0.05)$.

"Essential amino acid

Table 10: Seasonal variation of total amino acid contents in the dried sea tangle (S. japonica) cultured at Kijang area ${ }^{a}$

in this study was similar to the content observed in other seaweeds, comprising from $1 \%$ to $3 \%$ of dry matter $[20,21]$. The ash content varied from $14.3 \%$ to $18.4 \%$ in our samples. It has been reported that the ash content fluctuates depending on the species, geographical location, and season investigated $[22,23]$.

Component sugar compositions of Kijang and Wando samples were high in the following order: fucose, galactose, glucose, mannose, and so on. Polysaccharide of seaweed generally classified into cytoskeleton, intercellular mucoid, and storage polysaccharides, most of $2 \mathrm{M} \mathrm{HCl}$ hydrolyzed polysaccharide from $S$. japonica in this study originated from storage polysaccharide [24]. In the Kijang and Wando samples, there was a variation in the sugar content depending on culture period $(\mathrm{P}<0.05)$. Galactose content of Kijang samples were higher than that of Wando in the all culture period $(\mathrm{p}<0.05)$.

Major fatty acid of Kijang and Wando samples is myristic acid (14:0), palmitic acid (16:0), oleic acid (18:1), linoleic acid (18:2), 
Citation: Hwang JH, Kim NG, Woo HC, Rha SJ, Kim SJ, et al. (2014) Variation in the Chemical Composition of Saccharina Japonica with Harvest Area and Culture Period. J Aquac Res Development 5: 286. doi:10.4172/2155-9546.1000286

Page 6 of 7

\begin{tabular}{|c|c|c|c|c|c|c|}
\hline \multirow{2}{*}{ Amino acid } & \multicolumn{6}{|c|}{ Culture period } \\
\hline & Feb & Mar & Apr & May & Jun & Jul \\
\hline Aspatic acid & $1311.08 \pm 30.09^{a}$ & $1370.98 \pm 38.01^{a}$ & $1350.66 \pm 28.74^{a}$ & $1248.37 \pm 37.12^{b}$ & $1197.13 \pm 32.41^{\mathrm{c}}$ & $987.30 \pm 29.07^{d}$ \\
\hline Threonine* & $662.58 \pm 15.35^{a}$ & $686.76 \pm 15.65^{a}$ & $685.58 \pm 15.73^{a}$ & $593.84 \pm 11.69^{b}$ & $519.70 \pm 12.70^{c}$ & $403.39 \pm 3.13^{d}$ \\
\hline Serine & $622.40 \pm 14.98^{b}$ & $648.74 \pm 7.75^{a}$ & $643.88 \pm 12.84^{\mathrm{ab}}$ & $584.90 \pm 15.87^{c}$ & $539.02 \pm 13.64^{d}$ & $460.76 \pm 11.59^{e}$ \\
\hline Glutamic acid & $1490.24 \pm 37.35^{b}$ & $1563.56 \pm 30.46^{a}$ & $1541.39 \pm 40.47^{\mathrm{ab}}$ & $1490.61 \pm 30.71^{b}$ & $1257.52 \pm 8.65^{c}$ & $1170.02 \pm 18.63^{d}$ \\
\hline Proline & $518.72 \pm 12.20^{\mathrm{bc}}$ & $544.67 \pm 13.83^{a}$ & $536.49 \pm 10.88^{a b}$ & $511.15 \pm 8.81^{c}$ & $485.48 \pm 13.65^{d}$ & $384.76 \pm 7.06^{e}$ \\
\hline Glycine & $754.48 \pm 14.36^{b}$ & $787.88 \pm 16.64^{a}$ & $780.47 \pm 12.97^{a}$ & $686.67 \pm 14.51^{c}$ & $544.26 \pm 9.64^{d}$ & $526.76 \pm 12.53^{d}$ \\
\hline Alanine & $913.89 \pm 10.07^{b}$ & $955.28 \pm 19.29^{a}$ & $945.36 \pm 19.00^{\mathrm{ab}}$ & $867.07 \pm 24.20^{c}$ & $726.10 \pm 12.95^{d}$ & $718.06 \pm 19.23^{d}$ \\
\hline Cystine & N.D. & N.D. & N.D. & N.D. & N.D. & N.D. \\
\hline Valine $^{*}$ & $710.09 \pm 15.14^{b}$ & $743.50 \pm 16.17^{a}$ & $734.50 \pm 17.42^{\mathrm{ab}}$ & $581.42 \pm 15.16^{c}$ & $515.00 \pm 13.11^{d}$ & $458.48 \pm 11.91^{\mathrm{e}}$ \\
\hline Methionine & $295.23 \pm 6.77^{\mathrm{b}}$ & $312.42 \pm 7.13^{\mathrm{a}}$ & $305.21 \pm 6.73^{\mathrm{ab}}$ & $227.70 \pm 6.28^{c}$ & $211.71 \pm 6.35^{d}$ & $194.27 \pm 3.56^{\mathrm{e}}$ \\
\hline Isoleucine $^{*}$ & $572.64 \pm 15.91^{b}$ & $601.11 \pm 12.59^{a}$ & $592.24 \pm 14.98^{\mathrm{ab}}$ & $524.88 \pm 6.90^{c}$ & $510.59 \pm 6.78^{c}$ & $443.58 \pm 5.14^{d}$ \\
\hline Leucine* $^{*}$ & $1077.85 \pm 31.36^{b}$ & $1131.59 \pm 21.26^{a}$ & $1114.74 \pm 32.66^{\mathrm{ab}}$ & $941.52 \pm 28.54^{c}$ & $828.06 \pm 12.26^{d}$ & $626.19 \pm 19.45^{\mathrm{e}}$ \\
\hline Tyrosine* & $274.85 \pm 5.60^{\mathrm{b}}$ & $292.06 \pm 6.82^{\mathrm{a}}$ & $284.09 \pm 6.35^{\mathrm{ab}}$ & $224.07 \pm 4.65^{c}$ & $193.09 \pm 2.31^{e}$ & $205.54 \pm 5.27^{d}$ \\
\hline Phenylalanine* $^{*}$ & $640.38 \pm 14.90^{b}$ & $673.29 \pm 21.10^{a}$ & $662.28 \pm 15.18^{a b}$ & $551.35 \pm 13.22^{c}$ & $490.96 \pm 9.25^{d}$ & $368.61 \pm 7.66^{e}$ \\
\hline Histidine & $363.34 \pm 10.38^{b}$ & $368.28 \pm 8.41^{\mathrm{ab}}$ & $376.21 \pm 9.25^{a}$ & $313.23 \pm 8.08^{c}$ & $283.56 \pm 5.28^{d}$ & $241.80 \pm 4.95^{e}$ \\
\hline Lysine* & $642.57 \pm 20.25^{b}$ & $671.81 \pm 13.22^{\mathrm{a}}$ & $664.74 \pm 14.67^{a}$ & $542.88 \pm 11.71^{\mathrm{C}}$ & $493.55 \pm 12.33^{d}$ & $431.80 \pm 9.64^{\mathrm{e}}$ \\
\hline Arginine & $606.52 \pm 13.83^{a}$ & $627.61 \pm 14.36^{a}$ & $627.62 \pm 12.39^{a}$ & $529.72 \pm 14.13^{b}$ & $515.74 \pm 14.81^{b}$ & $458.52 \pm 13.99^{c}$ \\
\hline Total & $11456.88 \pm 261.03^{b}$ & $13776.53 \pm 366.56^{a}$ & $11845.47 \pm 251.11^{b}$ & $10498.01 \pm 363.89^{c}$ & $9862.18 \pm 7.70^{d}$ & $7609.83 \pm 206.81^{e}$ \\
\hline
\end{tabular}

aValues represent means \pm standard error $(n=3)$. Mean values in the same row followed by different letters differ significantly $(p<0.05)$.

*Essential amino acid

Table 11: Seasonal variation of total amino acid contents in the dried sea tangle (S. japonica) cultured at Wando area

a-linolenic acid (18:3), arachidonic acid (20:4), and lignoceric acid (24:0). Many researches on seaweed fatty acid composition have been reported [16,25-34], but there have been various fatty acid contents since which one is chosen for analysis among about 50 selling fatty acid standards. Moreover, fatty acid compositions of the seaweed are generally varied by analyzing its sampled part. In all the data, most fatty acid composition showed a variation with harvest area and culture period. Low fatty acids, such as lauric acid (12:0), stearic acid (18:0), and arachidic acid (20:0), showed different compositions on harvest area and culture period without tendency. These results are similar with previous report [16]. Linoleic acid, $\gamma$-linolenic acid, and arachidonic acid in Kijang samples and $\gamma$-linolenic acid, arachidonic acid, and lignoceric acid in Wando samples increased with culture period, whereas $a$-linolenic acid in Kijang samples and stearic acid in Wando samples decreased. Both Kijang and Wando samples decreased with culture period in saturates, while those of polyenes increased.

In the mineral contents of Kijang and Wando S. japonica samples, the results show that $S$. japonica is rich in $\mathrm{K}$ and $\mathrm{Na}$ with moderate amounts of $\mathrm{Ca}$ and $\mathrm{Mg}$ whereas $\mathrm{Cu}, \mathrm{Fe}, \mathrm{Mn}$, and $\mathrm{Zn}$ are present in small quantities.

Major amino acid of Kijang and Wando S. japonica samples are glutamic acid, aspartic acid, leucine, alanine, glycine, valine, phenylalanine, but cystine was not detected. Glutamic acid and aspartic acid occupied over $20 \%$ in the total amino acid. It is known that amino acid of seaweed is generally composed of high contents in neutral and acidic amino acids such as alanine, aspartic acid, glycine, and proline [24], but S. japonica contained low glycine and proline contents. Sulfur amino acid, cysteine and cysteine, was not detected, methionine, histidine, and tyrosine were included in small amount. The average percentages of essential amino acids (EAA) in Kijang and Wando S. japonica samples were $39.5 \%, 37.8 \%$, which is higher than the EAA requirement (32.3\%) suggested by the Food and Agriculture Organization [35]. The amino acid composition observed in this study was similar to previous studies [36], where the sum of the average percentage of three amino acids, glutamic acid (13.5\%), aspartic acid
(11.9\%), and alanine (7.9\%), comprised the greatest proportion $33.3 \%)$ of TAA composition. Noda [37] suggested that the former three amino acids (glutamic acid, aspartic acid, and alanine) might produce the flavors specific to Nori (Porphyra). TAA content decreased at the end of the culture period. This phenomenon has also been observed in other seaweeds such as Enteromorpha prolifera, C. fulvescens, and Codium fragile [38].

In conclusion, we have ascertained that the monthly nutritional composition of $S$. japonica affected by harvest area and culture period from February to July 2011. S. japonica in Kijang and Wando showed the highest crude protein content in February and the highest carbohydrate content in July. Fucose was the most abundant and galactose the second most abundant in the monosaccharide composition profiles. Significant increases of the major fatty acids in Kijang (C18:2 n-6 and C20:4n-6) and Wando (C18:3 n-6) were observed as the culture period progressed. The highest mineral content of both Kijang and Wando samples is potassium and followed by sodium, calcium, magnesium, and so on. In the total amino acid contents, Kijang samples increased from February to April but decreased from May to July, while Wando samples increased on March but decreased from April to July.

\section{Acknowledgment}

This study received financial support from the Ministry of Oceans and Fisheries, Republic of Korea.

\section{References}

1. Zemke-White WL, Ohno M (1999) World seaweed utilization: an endof- century summary. J Appl Phycol 11: 369-376.

2. Mohamed S, Hashim SN, Abdul Rahman H (2012) Seaweeds: A sustainable functional food for complementary and alternative therapy, Trends Food Sci Tech 23: 83-96

3. FAO (2009) FAO Yearbook of Fishery and Aquaculture statistics.

4. Park JI, Woo HC, Lee JH (2008) Production of bio-energy from marine algae: status and perspectives. Korean Chem Eng Res 46: 833-844.

5. Shin TS, Z Xue, YW Do, SI Jeong, HC Woo, et al. (2011) Chemical Properties of Sea Tangle (Saccharina. japonica) Cultured in the Different Depths of Seawater. Clean Technology 17: 395-405. 
Citation: Hwang JH, Kim NG, Woo HC, Rha SJ, Kim SJ, et al. (2014) Variation in the Chemical Composition of Saccharina Japonica with Harvest Area and Culture Period. J Aquac Res Development 5: 286. doi:10.4172/2155-9546.1000286

6. Patarra RF, Paiva L, Neto Al, Lima E, Baptista J (2011) Nutritional value of selected macroalgae. J Appl Phycol 23: 205-208.

7. Sun SM, Cho SY, Shin TS, Chung GH, Ahn CB, et al. (2012) Variation in the chemical composition of Capsosiphon fulvescens with area and during the harvest period. J Appl Phycol 24: 459-465.

8. Wheeler WN, Weindner W (1983) The effects of external inorganic nitrogen concentration on the metabolism of growth and activities of key carbon and nitrogen assimilatory enzymes of Laminaria saccharina(Phaeophyta) in culture. J Phycol 19: 91-96.

9. Ohno M, Crichley AT (1997) Seaweed cultivation and marine ranching

10. Cosson J (1973) Action de la temperature et de la lumiere sur le de velopment du gametophyte de la Laminaria digitata(L.) Lam.(Pheophycee, Laminariales).

11. Kang RS, Koh CH (1999) Germination and Growth of Laminaria japonica (Phaeophyta) Microscopic Stages under Different Temperatures and Photon Irradiances. J. Korean Fish Soc 32: 438-443.

12. AOAC (1995) Official Methods of Analysis

13. Amza T, Amadou I, Kamara MT, Zhu K, Zhou H (2010) Chemical and nutrient analysis of gingerbread plum (Neocarya macrophylla) seeds. Adv J Food Sci Technol 2: 191-195.

14. Chaplin MF, Kennedy JF (1994) Carbohydrate Analysis: A Practical Approach. Oxford University Press, Oxford, New York.

15. Iverson SJ, Lang SLC, Cooper MH (2011) Comparison of the Bligh and Dyer and Folch methods for total lipid determination in a broad range of marine tissue. Lipids 36: 1283-1287.

16. Khotimcheno SV, Kulikova IV (2000) Lipids of different parts of the Lamina of Laminaria japonica Aresch. Botanica Marina 43: 87-91.

17. Rosemberg G, Ramus J (1982) Ecological growth strategies in the seaweeds Gracilaria follifera and Ulva sp.: soluble nitrogen and reserve carbohydrates. Mar Biol 66: 251-259.

18. Shin DM, SR An, SK In, JG Koo (2013) Seasonal Variation in the Dietary Fiber Amino Acid and Fatty Acid Contents of Porphyra yezoensis. Kor J Fish Aquat Sci 46: 337-342.

19. Rotem A, Roth-Bejeranu N, Arad SM (1986) Effect of controlled environmental conditions on starch and agar contents of Gracilaria sp. J Phycol 22: 117-121.

20. Hwang EK, Amano H, Park CS (2008) Assessment of the nutritional value of Capsosiphon fulvescens (Chlorophyta): developing a new species of marine macroalgae for cultivation in Korea. J Appl Phycol 20: 147-151.

21. Manivannan K, Thirumaran G, Devi GK, Anantharaman P, Balasubramanian T (2009) Proximate composition of different group of seaweeds from Vedalai coast waters (Gulf of Mannar): southeast coast of India. Middle-East J Sci Res 4: 72-77.
22. Ruperez P (2002) Mineral content of edible marine seaweeds. Food Chem 78: 23-26.

23. Kaehler S, Kennish R (1996) Summer and winter comparisons in the nutritional value of marine macroalgae from Hong Kong. Bot Mar 39: 11-17.

24. Park YH, Jang DS, Kim SB (1997) Processing of the sea food. Hyungsul Press, Seoul, Korea.

25. Choe SN, Choi KJ (2000) Fatty Acid Compositions of Sea Algaes in The Southern Sea Coast of Korea. Kor J Food Nutr 15: 58-63.

26. Dembitsky VM, Rosentsvet OA, Pechenkina EE (1990) Glycolipids, Phospholipids and Fatty Acids of Brown Algae Species. Phytochem 29: 34173421

27. Fleurence J, Gutbier G, Mabeau S, Leray C (1994) Fatty Acids from 11 Marine Macroalgae of The French Brittany Coast. J Appl Phycol 6: 527-532.

28. Harwood JL (1980) Plant Acyl Lipids: Structure, Distribution and Analysis. Academic Press, New York.

29. Hayashi K, Kida S, Kato K, Yamada M (1974) Component Fatty Acids of Acetone-Soluble Lipids of 17 Species of Marine Benthic Algae. Nipp Suis Gak 40: $609-617$

30. Jamieson JM, Reid EH (1972) The Component Fatty Acids of Some Marine Algal Lipids. Phytochem 11: 1423-1432.

31. Kaneniwa M, Itabashi J, Takagi T (1987) Unusual 5-olefinic Acids in the Lipids of Algae from Japanese Waters. Nipp Suis Gak 53: 861-866.

32. Kato M, Ariga N (1983) Studies on Lipids of Marine Algae: Sterol and Fatty Acid Composition of Marine Algae. GifuDaigaku 18: 53-55.

33. Kim M, Dubacq JP, Thomas JK, Giraud G (1996) Seasonal Variations of Triacylglycerols and Fatty Acids in Fucus serratus. Phytochem 43: 49-55.

34. Pohl P, Zurheide F (1979) Fatty Acids and Lipids of Marine Algae and the Control of Their Biosynthesis by Environmental Factor. Pharma Science, Walter de Gruyter, New York.

35. FAO/WHO (1973) Energy and protein requirements.

36. Shin TS, Xue Z, Do YW, Jeong SI, Woo HC, et al. (2011) Chemical Properties of Sea Tangle (Saccharina. japonica) Cultured in the Different Depths of Seawater. Clean Technol 17: 395-405.

37. Noda N (1993) Health benefits and nutritional properties of nori. J Appl Phycol 5: $255-258$

38. Jung KJ, Jung CH, Pyeun JH, Choi YJ (2005) Changes of food components in Mesangi (Capsosiphon fulvescens), Gashiparae (Enteromorpha prolifera), and Cheonggak (Codium fragile) depending on harvest times. J Korean Soc Food Sci Nutr 34: 687- 693. 